\title{
ANTI-INVARIANT SUBMANIFOLDS SATISFYING A CERTAIN CONDITION ON NORMAL CONNECTION
}

\author{
By IKuo IshiHara
}

\section{$\S 1$. Introduction.}

In a previous paper [3] the present author studied anti-invariant submanifolds of a $(2 m+1)$-dimensional Sasakian manifold $\bar{M}$ with structure $(\phi, \xi, \eta, \bar{g})$ when the structure vector field $\xi$ is tangent to the submanifolds everywhere.

An $n$-dimensional Riemannian manifold $M$ isometrically immersed in $\bar{M}$ is said to be anti-invariant in $\bar{M}$ if $\phi T_{x}(M) \subset T_{x}(M)^{\perp}$ for each point $x$ of $M$, where $T_{x}(M)$ and $T_{x}(M)^{\perp}$ denote respectively the tangent and the normal spaces to $M$ at $x$. Thus, for any vector $X$ tangent to $M, \phi X$ is normal to $M$ because of the definition given above. $\phi$ is necessarily of rank $2 m$ and hence $n \leqq m+1$.

The purpose of the present paper is to study $n$-dimensional anti-invariant submanifolds normal to the structure vector field $\xi$ of a $(2 m+1)$-dimensional Sasakian manifold $\bar{M}$. If a submanifold $M$ of $\bar{M}$ is normal to the structure vector field $\xi$, then $M$ is anti-invariant in $\bar{M}$ as a consequence of Lemma 3.1. So, in this paper, we mean, by an anti-invariant submanifold $M$ of a Sasakian manifold $\bar{M}$, a submanifold $M$ normal to the structure vector field $\xi$ of a Sasakian manifold $\bar{M}$.

\section{$\S 2$. Sasakian manifolds.}

First, we would like to recall definitions and some fundamental properties of Sasakian manifolds. Let $\bar{M}$ be a $(2 m+1)$-dimensional differentiable manifold of class $C^{\infty}$ and $\phi, \xi, \eta$ be a tensor field of type $(1,1)$, a vector field, a 1 -form on $\bar{M}$ respectively such that

$$
\phi^{2}=-I+\eta \otimes \xi, \quad \phi \xi=0, \quad \eta(\phi X)=0, \quad \eta(\xi)=1
$$

for any vector field $X$ on $\bar{M}$, where I denotes the identity tensor of type $(1,1)$. Then $\bar{M}$ is said to admit an almost contact structure $(\phi, \xi, \eta)$ and called an almost contact manifold. The almost contact structure is said to be normal if

$$
N+d \eta \otimes \xi=0
$$

Received July 5, 1978 
where $N$ denotes the Nijenhuis tensor formed with $\phi$. If there is given in $\bar{M}$ a Riemannian metric $\bar{g}$ satisfying

$$
\bar{g}(\phi X, \phi Y)=\bar{g}(X, Y)-\eta(X) \eta(Y), \quad \eta(X)=\bar{g}(X, \xi)
$$

for any vector fields $X$ and $Y$ on $\bar{M}$, then the set $(\phi, \xi, \eta, \bar{g})$ is called a almost contact metric structure and $\bar{M}$ an almost contact metric manifold. If

$$
d \eta(X, Y)=\bar{g}(\phi X, Y)
$$

for any vector fields $X$ and $Y$ on $\bar{M}$, then the almost contact metric structure is called a contact metric structure. If the structure is moreover normal, then the contact metric structure is called a Sasakian structure and $\bar{M}$ a Sasakian manıfold. As is well known, in a Sasakian manifold $\bar{M}$ with structure $(\phi, \xi, \eta, \bar{g})$ the equations

$$
\bar{\nabla}_{X} \xi=\phi X, \quad\left(\bar{\nabla}_{X} \phi\right) Y=-\bar{g}(X, Y)+\eta(Y) X
$$

are established for any vector fields $X$ and $Y$ on $\bar{M}$, where $\bar{\nabla}$ denotes the operator of covariant differentiation with respect to $\bar{g}$.

A plane section $\sigma$ in the tangent space $T_{x}(\bar{M})$ of a Sasakian manifold $\bar{M}$ at $x$ is called a $\phi$-section if it is spanned by vectors $X$ and $\phi X$, where $X$ is assumed to be orthogonal to $\xi$. The sectional curvature $K(\sigma)$ with respect to a $\phi$-section $\sigma$ is called a $\phi$-sectronal curvature. When the $\phi$-sectional curvature $K(\sigma)$ is independent of the $\phi$-section $\sigma$ at each point of $\bar{M}$, as is well known, the function $K(\sigma)$ defined in $\bar{M}$ is necessarily a constant $c$. A Sasakian manifold $\bar{M}$ is called a Sasakıan space form and denoted by $\bar{M}(c)$ if it has constant $\phi$ sectional curvature $c$ (see [4]). The curvature tensor $K$ of a Sasakian space form $\bar{M}(c)$ is given by

$$
\begin{aligned}
K(X, Y) Z= & \frac{1}{4}(c+1)(\bar{g}(Y, Z) X-\bar{g}(X, Z) Y)-\frac{1}{4}(c-1)(\eta(Y) \eta(Z) X \\
& -\eta(X) \eta(Z) Y+\bar{g}(Y, Z) \eta(X) \xi-\bar{g}(X, Z) \eta(Y) \xi \\
& -\bar{g}(\phi Y, Z) \phi X+\bar{g}(\phi X, Z) \phi Y+2 \bar{g}(\phi X, Y) \phi Z)
\end{aligned}
$$

EXAMPLE 1. Let $S^{2 n+1}$ be a $(2 n+1)$-dimensional unit sphere, i. e.,

$$
S^{2 n+1}=\left\{z \in C^{n+1}:|z|=1\right\},
$$

where $C^{n+1}$ is a complex $(n+1)$-space. For any point $z \in S^{2 n+1}$, we put $\xi=J z, J$ being the complex structure of $C^{n+1}$. Considering the orthogonal projection

$$
\pi: T_{z}\left(C^{n+1}\right) \longrightarrow T_{z}\left(S^{2 n+1}\right),
$$

at each point $z$ in $S^{2 n+1}$ and putting $\phi=\pi \circ J$, we have a Sasakian structure $(\phi, \xi, \eta, g)$ on $S^{2 n+1}$, where $\eta$ is a 1 -form dual to $\xi$ and $g$ the standard metric tensor field on $S^{2 n+1}$. Obviously, $S^{2 n+1}$ is of constant $\phi$-sectional curvature 1 . 
EXAMPLE 2. Let $E^{2 n+1}$ be a Euclidean space with cartesian coordinates $\left(x^{1}, \cdots, x^{n}, y^{1}, \cdots, y^{n}, z\right)$. Then a Sasakian structure on $E^{2 n+1}$ is defined by $\phi, \xi, \eta$ and $g$ such that

$$
\begin{gathered}
\xi=(0, \cdots, 0,2), \quad 2 \eta=\left(-y^{1}, \cdots,-y^{n}, 0, \cdots, 0,1\right), \\
\left(g_{A B}\right)=\left(\begin{array}{ccc}
\frac{1}{4}\left(\delta_{\imath \jmath}+y^{2} y^{j}\right) & 0 & -\frac{1}{4} y^{\imath} \\
0 & \frac{1}{4} \delta_{\imath \jmath} & 0 \\
-\frac{1}{4} y^{\jmath} & 0 & \frac{1}{4}
\end{array}\right), \\
\left(\phi_{B}^{A}\right)-\left(\begin{array}{ccc}
0 & \delta_{\jmath}^{\imath} & 0 \\
-\delta_{J}^{2} & 0 & 0 \\
0 & y^{\jmath} & 0
\end{array}\right) .
\end{gathered}
$$

Then $E^{2 n+1}$ with such a structure $(\phi, \xi, \eta, g)$ is of constant $\phi$-sectional curvature -3 and denoted by $E^{2 n+1}(-3)$.

\section{$\S 3$. Fundamental properties.}

Let $\bar{M}^{2 m+1}$ be a Sasakian manifold of dimension $2 m+1$ with structure $(\phi, \xi, \eta, \bar{g})$. An $n$-dimensional Riemannian manifold $M$ isometrically immerssed in $\bar{M}^{2 m+1}$ is said to be antı-invariant in $\bar{M}^{2 m+1}$ if $\phi T_{x}(M) \subset T_{x}(M)^{\perp}$ for each point $x$ of $M$. Throughout the paper, we now restrict ourselves only to anti-invariant submanifolds of a Sasakian manifold such that the structure vector field $\xi$ of the ambient manifold is normal to the submanifolds.

Let $g$ be the induced metric tensor field of $M$. We denote by $\bar{\nabla}$ (resp. $\nabla$ ) the operator of covariant differentiation with respect to $\vec{g}$ (resp. $g$ ). Then the Gauss and Weingarten formulas are given respectively by

$$
\bar{\nabla}_{X} Y=\nabla_{X} Y+B(X, Y), \quad \bar{\nabla}_{X} N=-A_{N}(X)+D_{X} N
$$

for any vector fields $X, Y$ tangent to $M$ and any vector field $N$ normal to $M$, where $D$ is the operator of covariant differentiation with respect to the liner connection induced in the normal bundle. Both $A$ and $B$ are called the second fundamental form of $M$. They satisfy $\bar{g}(B(X, Y), N)=g\left(A_{N}(X), Y\right)$.

First of all, we prove

LEMMA 3.1. ([2,5]) Let $M$ be an n-dimensional submanifold of a Sasakian manifold $M^{2 m+1}$. If the structure vector field $\xi$ of the ambient manifold is normal to $M$ everywhere, then $M$ is an antz-invaruant submanfold of $\bar{M}^{2 m+1}$ and $n \leqq m$. 
Proof. Since the structure vector field $\xi$ is normal to $M$ everywhere, we have

$$
\bar{g}(\phi X, Y)=\bar{g}\left(\bar{\nabla}_{X} \xi, Y\right)=g\left(-A_{\xi}(X), Y\right)+\bar{g}\left(D_{X} \xi, Y\right)=-g\left(A_{\xi}(X), Y\right)
$$

for any vector fields $X$ and $Y$ tangent to $M$. Since $A_{\xi}$ is symmetric and $\phi$ is skew-symmetric, we have $A_{\xi}=0$ and $\phi X$ is normal to $M$. Thus $M$ is antiinvariant and $n \leqq m$.

Throughout the paper, by an anti-invariant submanifold $M$ of a Sasakian manifold $\bar{M}^{2 m+1}$, we mean a submanifold $M$ such that the structure vector field $\xi$ of the ambient manifold is normal to $M$.

We choose a local field of orthonormal frames $e_{1}, \cdots, e_{n} ; e_{n+1}, \cdots, e_{m} ; e_{0^{*}}=$ $\xi, e_{1^{*}}=\phi e_{1}, \cdots, e_{n^{*}}=\phi e_{n} ; e_{(n+1) *}=\phi e_{n+1}, \cdots, e_{m^{*}}=\phi e_{m}$ in $\bar{M}^{2 m+1}$ in such a way that $e_{1}, \cdots, e_{n}$ are along $M$ tangent to $M$. Taking such a field of frames of $\bar{M}^{2 m+1}$, we denote the dual coframes by $\omega^{1}, \cdots, \omega^{n} ; \omega^{n+1}, \cdots, \omega^{m} ; \omega^{0^{*}}=\eta, \omega^{1^{*}}, \cdots, \omega^{n^{*}}$; $\omega^{(n+1)^{*}}, \cdots, \omega^{m^{*}}$. Unless otherwise stated, let the range of indices be as follows:

$$
\begin{aligned}
& A, B, C, D=1, \cdots, m, 0^{*}, 1^{*}, \cdots, m^{*}, \\
& \imath, \jmath, k, l, s, t=1, \cdots, n, \\
& a, b, c, d=n+1, \cdots, m, 0^{*}, 1^{*}, \cdots, m^{*}, \\
& p, q, r=n+1, \cdots, m, 1^{*}, \cdots, m^{*}, \\
& \lambda, \mu, \nu=n+1, \cdots, m, 0^{*},(n+1)^{*}, \cdots, m^{*}, \\
& x, y, z=n+1, \cdots, m,(n+1)^{*}, \cdots, m^{*}, \\
& \alpha, \beta, \gamma=n+1, \cdots m,
\end{aligned}
$$

and use the so-called summation convention for these systems of indices. Then the structure equations of the Riemannian manifold $\bar{M}^{2 m+1}$ are given by

$$
\begin{gathered}
d \omega^{A}=-\omega_{B}^{A} \wedge \omega^{B}, \quad \omega_{B}^{A}+\omega_{A}^{B}=0, \\
d \omega_{B}^{A}=-\omega_{C}^{A} \wedge \omega_{B}^{C}+\Phi_{B}^{A}, \quad \Phi_{B}^{A}=\frac{1}{2} K_{B C D}^{A} \omega^{C} \wedge \omega^{D},
\end{gathered}
$$

where $K_{B C D}^{A}$ are components of the curvature tensor of $\bar{M}^{2 m+1}$ with respect to $\left\{e_{A}\right\}$ and $\omega_{B}^{A}$ satisfy

$$
\begin{array}{llll}
\omega_{j}^{2}=\omega_{j^{*}}^{i^{*}}, & \omega_{J}^{i^{*}}=\omega_{i}^{j^{*}}, & \omega^{2}=\omega_{0^{*}}^{i^{*}}, & \omega^{\imath^{*}}=-\omega_{0^{*}}^{2}, \\
\omega_{\beta}^{\alpha}=\omega_{\beta^{*}}^{\alpha^{*}}, & \omega_{\beta}^{\alpha^{*}}=\omega_{\alpha}^{\beta^{*}}, & \omega^{\alpha}=\omega_{0^{*}}^{\alpha^{*}}, & \omega^{\alpha^{*}}=-\omega_{0^{*}}^{\alpha}, \\
\omega_{\alpha}^{2}=\omega_{\alpha^{*}}^{2^{*}}, & \omega_{\alpha}^{2^{*}}=\omega_{i}^{\alpha^{*}} . & &
\end{array}
$$

Thus we have along $M$

$$
\omega^{a}=0,
$$

which implies $0=d \omega^{a}=-\omega_{\imath}^{a} \wedge \omega^{2}$ along $M$. Thus, by Cartan's lemma, we obtain along $M$ 


$$
\omega_{i}^{a}=h_{\imath j}^{a} \omega^{j}, \quad h_{\imath j}^{a}=h_{j i}^{a},
$$

which imply the following structure equations of the submanifold $M$;

$$
\begin{gathered}
d \omega^{2}=-\omega_{j}^{\imath} \wedge \omega^{\jmath}, \quad \omega_{j}^{2}+\omega_{\iota}^{\jmath}=0, \\
d \omega_{j}^{2}=-\omega_{k}^{l} \wedge \omega_{j}^{k}+\Omega_{\jmath}^{\imath}, \quad \Omega_{j}^{\imath}=\frac{1}{2} R_{j k l}^{\imath} \omega^{k} \wedge \omega^{l}, \\
R_{j k l}^{\imath}=K_{j k l}^{i}+\sum_{a}\left(h_{i k}^{a} h_{\jmath l}^{a}-h_{\iota l}^{a} h_{j k}^{a}\right), \\
d \omega_{l}^{a}=-\omega_{c}^{a} \wedge \omega_{b}^{a}+\Omega_{b}^{a}, \quad \Omega_{b}^{a}=\frac{1}{2} R_{b k l}^{a} \omega^{k} \wedge \omega^{l}, \\
R_{b k l}^{a}=K_{b k l}^{a}+\sum_{\imath}\left(h_{\imath k}^{a} h_{\imath l}^{b}-h_{i l}^{a} h_{\imath k}^{b}\right),
\end{gathered}
$$

where $R_{j k l}^{\imath}$ are components of the curvature tensor of $M$ with respect to $\left\{e_{\imath}\right\}$ and $R_{b k l}^{a}$ components of the curvature tensor of the normal bundle with respect to $\left\{e_{i}\right\}$ and $\left\{e_{a}\right\}$. The equations (3.8) and (3.10) are called respectively the equations of Gauss and those of Ricci for the submanifold $M$. The forms $\left(\omega_{j}^{i}\right)$ define the Riemannian connection of $M$ and the forms $\left(\omega_{b}^{a}\right)$ define the connection induced in the normal bundle of $M$.

From (3.3), (3.4) and (3.5) we have along $M$

$$
h_{\jmath k}^{2}=h_{k i}^{j}=h_{\imath \jmath}^{k}, \quad h_{\imath j}^{0^{*}}=0,
$$

where we donote $h_{j k}^{i *}$ simply by $h_{j k}^{2}$.

The second fundamental form $h_{\imath j}^{a} \omega^{2} \omega^{j} e_{a}$ is sometimes denoted by its components $h_{\imath \jmath}^{a}$. If the second fundamental form vanishes identically, i. e., $h_{\imath j}^{a}=0$ for all indices, then the submanifold is as usual said to be totally geodesic. If $h_{\imath \jmath}^{a}$ have the form $h_{\imath j}^{a}=\frac{1}{n}\left(\sum_{k} h_{k k}^{a}\right) \delta_{i \jmath}$ for a fixed index $a$, then the submanifold is said to be umbilical with respect to the normal vector $e_{a}$. If the submanifold $M$ is umbical with respect to all $e_{a}$, then $M$ is said to be totally umbilical. The vector field $\frac{1}{n}\left(\sum_{k} h_{k k}^{a} e_{a}\right)$ normal to $M$ is called the mean curvature vector of $M$. The submanifold $M$ is said to be minmmal if its mean curvature vector vanishes identically, i. e., $\sum_{k} h_{k k}^{a}=0$ for all $a$. We define the covariant derivative $h_{\imath \jmath k}^{a}$ of $h_{\imath \jmath}^{a}$ by

$$
h_{\imath \jmath k}^{a} \omega^{k}=d h_{\imath \jmath}^{a}-h_{l_{\jmath}}^{a} \omega_{\imath}^{l}-h_{i l}^{a} \omega_{j}^{l}+h_{\imath \jmath}^{b} \omega_{b}^{a} .
$$

If $h_{\imath j k}^{a}=0$ for all indices, the second fundamental form of $M$ is said to be parallel. If the mean curvature vector of $M$ is parallel with respect to the connection in the normal bundle, then the mean curvature vector of $M$ is said to be parallel. From (3.3), (3.4), (3.11) and (3.12), we obtain

$$
h_{\imath \jmath k}^{0 *}=-h_{\imath \jmath}^{k} .
$$


Thus, we have

LEMma 3.2. ([6]) Let $M$ be an n-dimensional anti-invariant submanifold of a Sasakian manifold $\bar{M}^{2 n+1}$. If the second fundamental form of $M$ is parallel, then $M$ is totally geodesic.

Using (3.13), we obtain

$$
\sum_{k} h_{k k \imath}^{0^{*}}=-\sum_{k} h_{k k}^{2}
$$

Thus, we have

LEMMA 3.3. ([7]) Let $M$ be an n-dimensional antr-invariant submanifold of a Sasakian manifold $\bar{M}^{2 n+1}$. If the mean curvature vector of $M$ is parallel, then $M$ is munmal.

Because of Lemmas 3.2 and 3.3, the conditions that the second fundamental form is parallel and that the mean curvature vector is parallel are not interesting for anti-invariant submanifolds, when $m=n$. Therefore we shall now introduce some new notions as follows. On an anti-invariant submanifold $M$ of a Sasakian manifold $\bar{M}^{2 m+1}$, if $h_{\imath j k}^{p}=0$ for all indices, then we say that the second fundamental form of $M$ is $\eta$-parallel. If $\sum_{k} h_{k k \imath}^{p}=0$ for all indices $\imath$ and $p$, then the mean curvature vector said to be $\eta$-parallel.

We now define the Laplacian $\Delta h_{\imath \jmath}^{a}$ of $h_{\imath \jmath}^{a}$ by

$$
\Delta h_{\imath \jmath}^{a}=\sum_{k} h_{\imath j k k}^{a}
$$

where we have defined $h_{\imath j k l}^{a}$ by

$$
h_{\imath j k l}^{a} \omega^{l}=d h_{\imath \jmath k}^{a}-h_{l j k}^{a} \omega_{i}^{l}-h_{\imath l k}^{a} \omega_{j}^{l}-h_{\imath j l}^{a} \omega_{k}^{l}+h_{\imath \jmath k}^{b} \omega_{b}^{a} .
$$

We shall establish a formula containing the Laplacian of $h_{\imath \jmath}^{a}$. In the sequel the second fundamental form of $M$ is assumed to satisfy the equation of Codazzi type, i. e.,

$$
h_{i j k}^{a}-h_{i k \jmath}^{a}=0 .
$$

Then, from (3.16), we have

$$
h_{\imath j k l}^{a}-h_{\imath j k l}^{a}=h_{t j}^{a} R_{\imath k l}^{t}+h_{i t}^{a} R_{j k l}^{t}-h_{\imath j}^{b} R_{b k l}^{a} .
$$

On the other hand, (3.15) and (3.17) imply

$$
\Delta h_{\imath j}^{a}=\sum_{k} h_{\imath j k k}^{a}=\sum_{k} h_{k \imath \jmath k}^{a} .
$$

From (3.17), (3.18) and (3.19), we obtain

$$
\Delta h_{\imath j}^{a}=\sum_{k}\left(h_{k k \imath j}^{a}+h_{k t}^{a} R_{\imath j k}^{t}+h_{t \imath}^{a} R_{k j k}^{t}-h_{k \imath}^{b} R_{b j k}^{a}\right) .
$$


Therefore for any submanifold $M$ satisfying the equation (3.17) of Codazzi type we have the formula

$$
\begin{aligned}
\sum_{a, \imath, \jmath} h_{\imath j}^{a} \Delta h_{\imath j}^{a}= & \sum_{a, \imath, j, k}\left(h_{\imath j}^{a} h_{k k \imath \jmath}^{a}+h_{\imath j}^{a} h_{k t}^{a} R_{\imath \jmath k}^{t}\right. \\
& \left.+h_{\imath j}^{a} h_{\iota \imath}^{a} R_{k j k}^{t}-h_{\imath j}^{a} h_{k \imath}^{b} R_{b j k}^{a}\right) .
\end{aligned}
$$

If the ambient manifold $\bar{M}^{2 m+1}$ is of constant $\phi$-sectional curvature $c$, then the Riemannian curvature tensor of $\bar{M}^{2 m+1}$ has the form

$$
\begin{aligned}
K_{B C D}^{A}= & \frac{1}{4}(c+3)\left(\delta_{A C} \delta_{B D}-\delta_{A D} \delta_{B C}\right)+\frac{1}{4}(c-1)\left(\eta_{B} \eta_{C} \delta_{A D}\right. \\
& -\eta_{B} \eta_{D} \delta_{A C}+\eta_{A} \eta_{D} \delta_{B C}-\eta_{A} \eta_{C} \delta_{B D}+\phi_{A C} \phi_{B D} \\
& \left.-\phi_{A D} \phi_{B C}+2 \phi_{A B} \phi_{C D}\right),
\end{aligned}
$$

and the second fundamental form of $M$ satisfies the equation (3.17) of Codazzi type.

\section{$\S 4$. Normal connection.}

In this section we study the normal connection of an $n$-dimensional antiinvariant submanifold $M$ of a $(2 m+1)$-dimensional Sasakian space form $\bar{M}^{2 m+1}(c)$ when the structure vector field $\xi$ is normal to $M$. The curvature tensor of the normal connection of $M$ is assumed to have the form

$$
R_{b k l}^{a}=-\left(\delta_{a k^{*}} \delta_{b l^{*}}-\delta_{a l^{*}} \delta_{b k^{*}}\right) .
$$

LEMMA 4.1. Let $M$ be an n-dimensional antr-invariant submanfold of a Sasakian manifold $\bar{M}^{2 m+1}$. If the curvature tensor of the normal connnection of $M$ is of the form (4.1), then

$$
R_{j k l}^{\imath}=\sum_{x}\left(h_{\imath k}^{x} h_{j l}^{x}-h_{i l}^{x} h_{j k}^{x}\right) .
$$

Proof. (3.2) and (3.3) imply

$$
K_{j k l}^{i}=K_{j^{*} k l}^{i^{*}}+\left(\delta_{i k} \delta_{\jmath l}-\delta_{i l} \delta_{j k}\right) .
$$

Moreover, from (3.8), (3.10) and (3.11), we obtain

$$
\begin{aligned}
R_{j k l}^{\imath} & =K_{j k l}^{i}+\sum_{a}\left(h_{i k}^{a} h_{j l}^{a}-h_{i l}^{a} h_{j k}^{a}\right) \\
& =K_{j k l}^{i}+\sum_{t}\left(h_{t k}^{i} h_{t l}^{j}-h_{t l}^{i} h_{t k}^{j}\right)+\sum_{x}\left(h_{\imath k}^{x} h_{i l}^{x}-h_{i l}^{x} h_{j k}^{x}\right) \\
& =R_{i^{*} k l}^{j^{*}}+\left(\delta_{i k} \delta_{j l}-\delta_{i l} \delta_{j k}\right)+\sum_{x}\left(h_{i k}^{x} h_{j l}^{x}-h_{\imath l}^{x} h_{j k}^{x}\right),
\end{aligned}
$$

which proves Lemma 4.1. 
By (3.22) we obtain

$$
\begin{aligned}
& K_{j^{*} k l}^{\lambda}=0, \quad K_{j k l}^{\lambda}=0, \quad K_{\mu k l}^{\lambda}=0, \\
& K_{j^{*} k l}^{i *}=\frac{1}{4}(c-1)\left(\delta_{\imath k} \delta_{\jmath l}-\delta_{i l} \delta_{j k}\right) .
\end{aligned}
$$

If the curvature tensor of the normal connection of $M$ is of the form (4.1), then (3.10) and (4.5) imply

$$
\begin{aligned}
& \sum_{t}\left(h_{t k}^{x} h_{t l}^{\imath}-h_{t l}^{x} h_{t k}^{2}\right)=0, \quad \sum_{t}\left(h_{t k}^{x} h_{t l}^{y}-h_{t l}^{x} h_{t k}^{y}\right)=0, \\
& \sum_{t}\left(h_{t k}^{2} h_{t l}^{\jmath}-h_{t l}^{\imath} h_{t k}^{\jmath}\right)=-\frac{1}{4}(c+3)\left(\delta_{i k} \delta_{j l}-\delta_{\imath \iota} \delta_{\jmath k}\right) .
\end{aligned}
$$

Proposition 4.2. Let $M$ be an n-dimensional $(n>1)$ anti-invariant submanifold of a Sasakian space form $\bar{M}^{2 m+1}(c)$. If the curvature tensor of the normal connection of $M$ has the form (4.1) and $M$ is umbilical with respect to some $e_{t^{*}}$, then $c=-3$.

Proof. If $M$ is umbilical with respect to $e_{t^{*}}$, then the second fundamental form $h_{\imath \jmath}^{t}$ is of the form $h_{\imath \jmath}^{t}=\frac{1}{n}\left(\sum_{k} h_{k k}^{t}\right) \delta_{\imath \jmath}$. Thus we have

$$
\sum_{\imath}\left(h_{\imath k}^{t} h_{i l}^{s}-h_{\imath l}^{t} h_{\imath k}^{s}\right)=0 \text {. }
$$

From this and (4.7) we find $c=-3$.

For each fixed index $a$, we consider a symmetric $(n, n)$-matrix $A_{a}=\left(h_{\imath j}^{a}\right)$ composed of components of the second fundamental form.

LEMMA 4.3. Let $M$ be an n-dimensional antz-invariant submanfold of a Sasakian space form $\bar{M}^{2 m+1}(c)(c \neq-3)$. If the curvature tensor of the normal connectron of $M$ is of the form (4.1), then $M$ is umbilical with respect to all $e_{x}$.

Proof. From (4.6) we obtain $A_{x} A_{y}=A_{y} A_{x}$ and $A_{x} A_{1}=A_{1} A_{x}$ for all $x$ and $y$. Therefore we can choose a local field of orthonormal frames with respect to which $A_{1}$ and all $A_{x}$ are diagonal, i. e.,

$$
A_{1}=\left(\begin{array}{ccc}
h_{11}^{1} & & 0 \\
0 & \cdot & h_{n n}^{1}
\end{array}\right), \quad A_{x}=\left(\begin{array}{ccc}
h_{11}^{x} & & 0 \\
0 & & h_{n n}^{x}
\end{array}\right) .
$$

Putting $\imath=l$ and $k=l$ in the first equation of (4.6) and using (3.11) and (4.8), we find

$$
\left(h_{11}^{x}-h_{i i}^{x}\right) h_{i i}^{1}=0 \text {. }
$$

On the other hand, putting $\imath=k=1$ and $\jmath=l \neq 1$ in (4.7) and using (3.11) and (4.8), we have 


$$
\left(h_{11}^{1}-h_{\jmath j}^{1}\right) h_{\jmath \jmath}^{1}=-\frac{1}{4}(c+3) .
$$

Since $c \neq-3$, (4.10) implies that $h_{j j}^{1} \neq 0(\jmath=2, \cdots, n)$. From this fact and (4.9) we find that $h_{l l}^{x}=h_{\jmath j}^{x}$ for all $x$. Thus $M$ is umbilical with respect to all $e_{x}$. This proves Lemma 4.3.

LEMMA 4.4. Let $M$ be an n-dimensional antr-invariant submanfold of a Sasakian space from $\bar{M}^{2 m+1}(c)(c \neq-3)$. If the curvature tensor of the normal connection of $M$ is of the form (4.1), then

$$
R_{j k l}^{2}=\frac{1}{n^{2}} \sum_{x}\left(\operatorname{Tr} A_{x}\right)^{2}\left(\delta_{\imath k} \delta_{j l}-\delta_{i l} \delta_{j k}\right)
$$

Proof. Lemma 4.3 implies $h_{i j}^{x}=\frac{1}{n}\left(\operatorname{Tr} A_{x}\right) \delta_{\imath}$ for all $x$. Therefore (4.2) implies (4.11).

In the Lemma 4.4 , if $n \geqq 3$, then $\sum_{x}\left(\operatorname{Tr} A_{x}\right)^{2}$ is constant. Therefore we have

Proposition 4.5 Let $M$ be an $n$-dimensional ( $n \geqq 3$ ) antr-invarzant submanifold of a Sasakian space form $\bar{M}^{2 m+1}(c)(c \neq-3)$. If the curvature tensor of the normal connection of $M$ has the form (4.1), then $M$ is of constant curvature.

If $M$ is minimal, then $\operatorname{Tr} A_{x}=0$ for all $x$. Thus Lemma 4.4 implies immediately

Proposition 4.6. Let $M$ be an $n$-dimensional antr-invariant mmmal submanifold of a Sasakian space form $\bar{M}^{2 m+1}(c)(c \neq-3)$. If the curvature tensor of the normal connection of $M$ has the form (4.1), then $M$ is flat.

\section{$\S 5$. $\eta$-parallel mean curvature vector.}

Using the results obtain in the previous section, we have

THEOREM 1. Let $M$ be an $n$-dimensional ( $n \geqq 3)$ ant-invariant submanifold of a Sasakian space form $\bar{M}^{2 m+1}(c)(c \neq-3)$ with $\eta$-parallel mean curvature vector. If the curvature tensor of the normal connection of $M$ is of the form (4.1), then there is in $\bar{M}^{2 m+1}(c)$ a totally geodesic and invariant submanifold $\bar{M}^{2 n+1}(c)$ of dimension $2 n+1$ in such a way that $M$ is immersed $2 n \bar{M}^{2 n+1}(c)$ as a flat antzınvariant minımal submanfold.

Proof. First of all, $\sum_{a}\left(\operatorname{Tr} A_{a}\right)^{2}$ is constant because the mean curvature vector is $\eta$-parallel. Since $n \geqq 3, \sum_{x}\left(\operatorname{Tr} A_{x}\right)^{2}$ is constant. On the other hand, from (3.8), (3.22) and (4.11), we have 


$$
\frac{n-1}{n} \sum_{x}\left(\operatorname{Tr} A_{x}\right)^{2}=\frac{1}{4} n(n-1)(c+3)+\sum_{a}\left(\operatorname{Tr} A_{a}\right)^{2}-\sum_{a, \imath, \jmath}\left(h_{\imath \jmath}^{a}\right)^{2} .
$$

Therefore the square of the length of the second fundamental form of $M$ is constant, i. e., $\sum_{a, \imath, j}\left(h_{i, j}^{a}\right)^{2}$ is constant. From this we have

$$
\sum_{a, \imath, j, k}\left(h_{\imath j k}^{a}\right)^{2}+\sum_{a, \imath, \jmath} h_{\imath j}^{a} \Delta h_{\imath j}^{a}=\frac{1}{2} \Delta \sum_{a, \imath, \jmath}\left(h_{\imath j}^{a}\right)^{2}=0 .
$$

By assumption, (3.11) and (3.21), we have

$$
\begin{aligned}
\sum_{a, \imath, j} h_{\imath j}^{a} \Delta h_{\imath j}^{a}= & \sum_{a, \imath, j, k}\left(h_{\imath j}^{a} h_{k t}^{a} R_{\imath j k}^{t}+h_{\imath j}^{a} h_{\imath \imath}^{a} R_{k j k}^{t}\right) \\
& +\sum_{\imath, j, k}\left(h_{i i}^{k} h_{\jmath j}^{k}-\left(h_{\imath j}^{k}\right)^{2}\right) .
\end{aligned}
$$

Moreover substituting (4.11) into (5.3) and using (5.2), we obtain

$$
\begin{aligned}
\sum_{a, \imath, \jmath, k}\left(h_{\imath j k}^{a}\right)^{2}= & -\frac{1}{n^{2}} \sum_{x}\left(\operatorname{Tr} A_{x}\right)^{2} \sum_{a, \imath, \jmath}\left(n\left(h_{\imath \jmath}^{a}\right)^{2}-h_{i i}^{a} h_{j j}^{a}\right) \\
& -\sum_{\imath, \jmath, k}\left(h_{\imath i}^{k} h_{\jmath j}^{k}-\left(h_{\imath j}^{k}\right)^{2}\right) .
\end{aligned}
$$

From Lemma 4.3 and (3.13), we have

$$
\begin{aligned}
\sum_{p, \imath, \jmath, k}\left(h_{\imath j k}^{p}\right)^{2}= & -\frac{1}{n^{2}} \sum_{x}\left(\operatorname{Tr} A_{x}\right)^{2} \sum_{\imath, \jmath, k}\left(n\left(h_{\imath j}^{k}\right)^{2}-h_{\imath i}^{k} h_{j \jmath}^{k}\right)-\sum_{\imath, \jmath, k} h_{i i}^{k} h_{j j}^{k} \\
= & -\frac{1}{n^{2}} \sum_{x}\left(\operatorname{Tr} A_{x}\right)^{2} \sum_{k}\left(\sum_{i<j}\left(h_{i i}^{k}-h_{j j}^{k}\right)^{2}+n \sum_{\imath \neq j}\left(h_{\imath j}^{k}\right)^{2}\right) \\
& -\sum_{k}\left(\operatorname{Tr} A_{k^{*}}\right)^{2} .
\end{aligned}
$$

Since $c \neq-3$ by assumption, Proposition 4.2 implies $\sum_{i<j}\left(h_{i i}^{k}-h_{j j}^{k}\right)^{2}>0$. Thus (5.5) implies $\operatorname{Tr} A_{x}=0, \operatorname{Tr} A_{k^{*}}=0$ and $h_{\imath j k}^{p}=0$, that is, the second fundamental form is $\eta$-parallel. Lemma 4.4, $\operatorname{Tr} A_{x}=0, \operatorname{Tr} A_{k^{*}}=0$ and (3.11) mean that $M$ is flat and minimal. On the other hand, by Lemma 4.3, $\operatorname{Tr} A_{x}=0$ implies $A_{x}=0$ for all $x$. From (3.5) and $A_{x}=0$, we obtain $\omega_{i}^{x}=0$ and hence $\omega_{i^{*}}^{x}=0$ along $M$, by (3.3). Moreover, (3.3) and (3.4) imply $\omega_{0^{*}}^{x}=0$ along $M$. From the arguments above, taking account of a fundamental theorem in the theory of submanifolds, we see that $M$ is an anti-invariant submanifold immersed in some totally geodesic and $(2 n+1)$-dimensional submanifold $\bar{M}^{2 n+1}(c)$ of $\bar{M}^{2 m+1}(c)$ (see $\S 6$ in [3]). And the submanifold $\bar{M}^{2 n+1}(c)$ is invariant (see $\S 6$ in [3]). Thus Theorem 1 is proved.

In Theorem 1 , the case where $n=2$, i. e., where $M$ is 2-dimensional, is excluded. However, the same conclusions will be established even if $n=2$, provided that $M$ is compact. To establish this fact, we now prove 
THEOREM 2. Let $M$ be an n-dimensional antr-invariant submanifold of a Sasakian space form $\bar{M}^{2 m+1}(c)(c \neq-3)$ with $\eta$-parallel mean curvature vector and assumed to be compact. If the curvature tensor of the normal connection of $M$ is of the form (4.1), then $M$ is a flat anti-invariant minumal submanifold of a certain $(2 n+1)$-dimensional totally geodesic submanifold $\bar{M}^{2 n+1}(c)$ of $\bar{M}^{2 m+1}(c)$.

Proof. Since $M$ is compact, we have

$$
\int_{M} \sum_{a, \imath, j, k}\left(h_{\imath j k}^{a}\right)^{2} * 1=-\int_{M} \sum_{a, \imath, j} h_{\imath j}^{a} \Delta h_{\imath j}^{a} * 1,
$$

where $* 1$ denotes the volume element of $M$ (see (5.2)). Using this formula, we can prove Theorem 2 by a same way as taken to prove Theorem 1 .

We shall now consider the case where $c=-3$.

Proposition 5.1. Let $M$ be an n-dimensional ( $n \geqq 3$ ) ant-invariant submanfold of a Sasakian space form $\bar{M}^{2 m+1}(-3)$ with $\eta$-parallel mean curvature vector and the curvature tensor of the normal connection of $M$ be of the form (4.1). If $M$ is umbilical with respect to all $e_{x}$, then $M$ is a totally umbilical antr-invariant submanifold.

Proof. From (3.10), (3.22) and (4.1), we obtain

$$
\sum_{t}\left(h_{t k}^{a} h_{t l}^{b}-h_{t l}^{a} h_{t k}^{b}\right)=0 .
$$

Therefore we can choose a local field of orthonormal frames with respect to which all $A_{a}$ are simultaneously diagonal, i. e.,

$$
A_{a}=\left(\begin{array}{ccc}
h_{11}^{a} & & 0 \\
0 & \ddots & h_{n n}^{a}
\end{array}\right)
$$

Moreover, (3.11) implies that $h_{j k}^{\imath}=0$ unless $\imath=\jmath=k$. On the other hand, from the assumption and (4.2) we have the equation (4.11). Therefore the equation (5.5) holds and hence we have

$$
\sum_{p, \imath, j, k}\left(h_{\imath j k}^{p}\right)^{2}=-\sum_{k}\left(h_{k k}^{k}\right)^{2}\left(\frac{n-1}{n} \sum_{x}\left(\operatorname{Tr} A_{x}\right)^{2}+1\right) .
$$

Therefore we have $h_{k k}^{k}=0$, that is, $A_{k^{*}}=0$ for all $k$. Thus $M$ is totally umbilical.

Remark. In Proposition 5.1, the case where $n=2$, that is, where $M$ is 2 dimensional, is excluded. However, the same conclusions are established even if $n=2$, provided that $M$ is compact.

EXAMPLE 5.2. Let $J$ be the almost complex structure of the complex $(n+1)$ space $C^{n+1}$ given by 


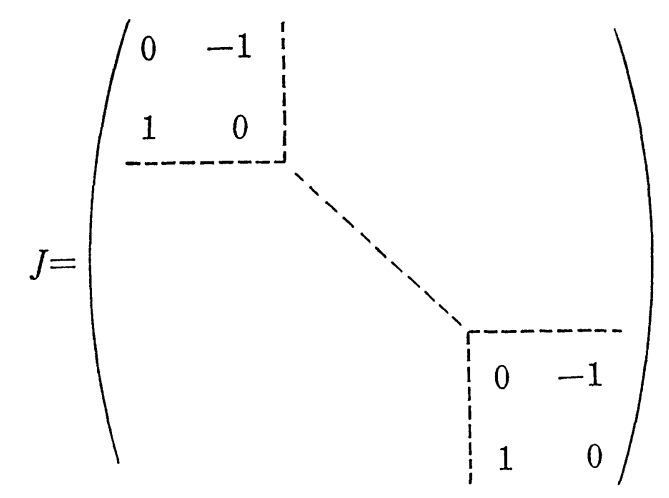

Let $S^{2 n+1}$ be a $(2 n+1)$-dimensional unit sphere in $C^{n+1}$ with standard Sasakian structure $(\phi, \xi, \eta, \bar{g})$. Let $S^{1}$ be a circle of radius 1 . Let us consider

$$
T^{n}=S^{1} \times \cdots \times S^{1} .
$$

Then we construct an isometric minimal immersion of $T^{n}$ into $S^{2 n+1}$ which is anti-invariant in the following way. Let $X: T^{n} \rightarrow S^{2 n+1}$ be a minimal immersion represented by

$$
X=\frac{1}{\sqrt{n+1}}=\left(\cos u^{1}, \sin u^{1}, \cdots, \cos u^{n}, \sin u^{n}, \cos u^{n+1}, \sin u^{n+1}\right),
$$

where we have put $u^{n+1}=-\left(u^{1}+\cdots+u^{n}\right)$. We may regard $X$ as a position vector of $S^{2 n+1}$ in $C^{n+1}$. The structure vector field $\xi$ of $S^{2 n+1}$, restricted to $T^{n}$, is then given by

$$
\xi=J X=\frac{1}{\sqrt{n+1}}\left(-\sin u^{1}, \cos u^{1}, \cdots,-\sin u^{n}, \cos u^{n},-\sin u^{n+1}, \cos u^{n+1}\right) .
$$

Putting $X_{i}=\partial X / \partial u^{\imath}$, we have

$$
X_{\imath}=\frac{1}{\sqrt{n+1}}\left(0, \cdots, 0,-\sin u^{2}, \cos u^{2}, 0, \cdots, 0, \sin u^{n+1},-\cos u^{n+1}\right),
$$

where $i=1, \cdots, n$. Thus $X_{\imath}, \imath=1, \cdots, n$, are linearly independent and $\eta\left(X_{\imath}\right)=0$ for $\imath=1, \cdots, n$. Therefore the immersion $X$ is anti-invariant.

The integral curves of the structure vector field $\xi$ are great circles $S^{1}$ in $S^{2 n+1}$ which are the fibres of the standard fibration $\pi: S^{2 n+1} \rightarrow C P^{n}$, where $C P^{n}$ is a complex projective space of complex dimension $n$ and of constant holomorphic sectional curvature 4 . Now we consider the following diagram:

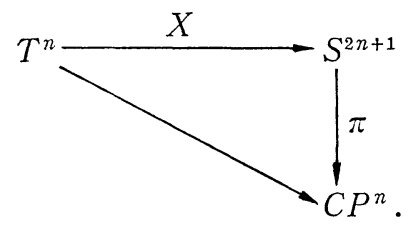


We easily see that $\left.\pi\right|_{X\left(T^{n}\right)}$ is one to one. Consequently $T^{n}$ is imbedded in $C P^{n}$ by $\pi \circ X$.

By Theorems 1, 2 and Example 5.2, we have

THEOREM 3. Let $M$ be an n-dimensional compact orientable anti-invariant submanifold of a Sasakian space form $S^{2 m+1}$ with $\eta$-parallel mean curvature vector. If the curvature tensor of the normal connection of $M$ is of the form (4.1), then $M$ is a torus $S^{1} \times \cdots \times S^{1}$ in some $S^{2 n+1}$ in $S^{2 m+1}$.

\section{BIBLIOGRAPHY}

[1] D. E. BLAIR, Contact manifolds in Riemannian geometry, Lecture Notes in Mathematics Vol 509 Springer Verlag, (1976).

[2] D. E. Blair AND K.Ogiue, Geometry of integral submanifolds of a contact distribution, Illinois J. Math., 19 (1975), 269-276.

[3] I. IshiHARA, Anti-invariant submanifolds of a Sasakian space form, Kodar Math. J., 2 (1979), 171-186.

[4] S. SASAKI, Almost contact manifolds, Lecture Note I, Tôhoku University, (1965).

[5] S. Yamaguchi and T. IKawa, On compact minimal C-totally real submanifolds, Tensor N.S., 29 (1975), 30-34.

[6] S. Yamaguchi, M. Kon and T. IKawa, On C-totally real submanifolds, J. Differential Geometry, 11 (1976) 59-64.

[7] K. YANO AND M. Kon, Anti-invariant submanifolds of Sasakian space forms II, J. Korean Math. Soc., 13 (1976), 1-14.

[8] K. Yano AND M. Kon, Anti-invariant submanifolds, Lecture Notes in Pure and Applied Mathematics Vol 21, Marcel Dekker Inc., New York, (1976).

[9] K. Yano, M. KON AND I. IshinARA, Ant1-invariant submanifolds with flat normal connection, to appear in J. Differential Geometry.

Tokyo Metropolitan Technical College

Higashi-ohi, Shinagawa-ku,

TOKYO, JAPAN 